\title{
Hawking-Unruh Hadronization and Strangeness Production in High Energy Collisions
}

\author{
Paolo Castorina ${ }^{1,2,3}$ and Helmut Satz ${ }^{4}$ \\ ${ }^{1}$ Dipartimento di Fisica ed Astronomia, Università di Catania, Via Santa Sofia 64, 95100 Catania, Italy \\ ${ }^{2}$ INFN Sezione di Catania, Via Santa Sofia 64, 95100 Catania, Italy \\ ${ }^{3}$ PH Department, TH Unit, CERN, 1211 Geneva 23, Switzerland \\ ${ }^{4}$ Fakultät für Physik, Universität Bielefeld, Germany \\ Correspondence should be addressed to Paolo Castorina; paolo.castorina@ct.infn.it
}

Received 8 January 2014; Accepted 16 April 2014; Published 11 May 2014

Academic Editor: Piero Nicolini

Copyright (C) 2014 P. Castorina and H. Satz. This is an open access article distributed under the Creative Commons Attribution License, which permits unrestricted use, distribution, and reproduction in any medium, provided the original work is properly cited. The publication of this article was funded by SCOAP S $^{3}$

\begin{abstract}
The thermal multihadron production observed in different high energy collisions poses many basic problems: why do even elementary, $e^{+} e^{-}$and hadron-hadron, collisions show thermal behaviour? Why is there in such interactions a suppression of strange particle production? Why does the strangeness suppression almost disappear in relativistic heavy ion collisions? Why in these collisions is the thermalization time less than $\simeq 0.5 \mathrm{fm} / \mathrm{c}$ ? We show that the recently proposed mechanism of thermal hadron production through Hawking-Unruh radiation can naturally answer the previous questions. Indeed, the interpretation of quark $(q)$ antiquark $(\bar{q})$ pairs production, by the sequential string breaking, as tunneling through the event horizon of colour confinement leads to thermal behavior with a universal temperature, $T \simeq 170 \mathrm{Mev}$, related to the quark acceleration, $a$, by $T=a / 2 \pi$. The resulting temperature depends on the quark mass and then on the content of the produced hadrons, causing a deviation from full equilibrium and hence a suppression of strange particle production in elementary collisions. In nucleus-nucleus collisions, where the quark density is much bigger, one has to introduce an average temperature (acceleration) which dilutes the quark mass effect and the strangeness suppression almost disappears.
\end{abstract}

\section{Introduction}

Hadron production in high energy collisions shows remarkably universal thermal features. In $e^{+} e^{-}$annihilation [1-3], in $p p$, in $p \bar{p}$ [4], and more general $h h$ interactions [3], as well as in the collisions of heavy nuclei [5-11], over an energy range from around $10 \mathrm{GeV}$ up to the $\mathrm{TeV}$ range; the relative abundances of the produced hadrons appear to be those of an ideal hadronic resonance gas at a quite universal temperature $T_{H} \approx 160-170 \mathrm{MeV}[12]$.

There is, however, one important nonequilibrium effect observed: the production of strange hadrons in elementary collisions is suppressed relative to an overall equilibrium. This is usually taken into account phenomenologically by introducing an overall strangeness suppression factor $\gamma_{s}<1$ [13], which reduces the predicted abundances by $\gamma_{s}, \gamma_{s}^{2}$, and $\gamma_{s}^{3}$ for hadrons containing one, two, or three strange quarks (or antiquarks), respectively. In high energy heavy ion collisions, strangeness suppression becomes less and disappears at high energies $[14,15]$.

The origin of the observed thermal behaviour has been an enigma for many years and there is a still ongoing debate about the interpretation of these results [16-24]. Indeed, in high energy heavy ion collisions multiple parton scattering could lead to kinetic thermalization, but $e^{+} e^{-}$or elementary hadron interactions do not readily allow such a description.

The universality of the observed temperatures, on the other hand, suggests a common origin for all high energy collisions, and it was recently proposed [25] that thermal hadron production is the QCD counterpart of HawkingUnruh (H-U) radiation $[26,27]$, emitted at the event horizon 
due to colour confinement. In the case of approximately massless quarks, the resulting hadronization temperature is determined by the string tension $\sigma$, with $T \simeq \sqrt{\sigma / 2 \pi} \simeq$ $170 \mathrm{Mev}$ [25]. Moreover in [28], it has been shown that strangeness suppression in elementary collisions naturally occurs in this framework without requiring a specific suppression factor. The crucial role here is played by the nonnegligible strange quark mass, which modifies the emission temperature for such quarks: in the Hawking-Unruh approach the temperature associated with strange particle production is different from that one of nonstrange particles. Although this conclusion has been obtained by a full analysis in the statistical hadronization model in [28], here we propose an elementary scheme of understanding strangeness suppression which, moreover, opens the possibility of explaining why this suppression disappears in heavy ion collisions where the large parton density in causally connected space-time region of hadronization produces a unique temperature for strange and nonstrange particles.

In particular, in the second section, after recalling the statistical hadronization model, one discusses the subsequent description in terms of $\mathrm{H}-\mathrm{U}$ radiation in QCD, including the dependence of the radiation temperature on the mass of the produced quark and the strangeness suppression in elementary collisions. In Section 3 the dynamical mechanism for strangeness enhancement (i.e., no suppression) in relativistic heavy ion scatterings is introduced giving a coarse grain evaluation of the Wroblewski factor [29], a parameter which counts the relative number of strange and nonstrange quarks and antiquarks. Section 4 is devoted to comments and conclusions.

\section{Hawking-Unruh Hadronization}

In this section we will recall the essentials of the statistical hadronization model and of the Hawking-Unruh analysis of the string breaking mechanism. For a detailed descriptions see $[3,25,28]$.

2.1. Statistical Hadronization Model. The statistical hadronization model assumes that hadronization in high energy collisions is a universal process proceeding through the formation of multiple colourless massive clusters (or fireballs) of finite spacial extension. These clusters are taken to decay into hadrons according to a purely statistical law: every multihadron state of the cluster phase space defined by its mass, volume, and charges is equally probable. The mass distribution and the distribution of charges (electric, baryonic, and strange) among the clusters and their (fluctuating) number are determined in the prior dynamical stage of the process. Once these distributions are known, each cluster can be hadronized on the basis of statistical equilibrium, leading to the calculation of averages in the microcanonical ensemble, enforcing the exact conservation of energy and charges of each cluster.

Hence, in principle, one would need the mentioned dynamical information in order to make definite quantitative predictions to be compared with data. Nevertheless, for
Lorentz-invariant quantities such as multiplicities, one can introduce a simplifying assumption and thereby obtain a simple analytical expression in terms of a temperature. The key point is to assume that the distribution of masses and charges among clusters is again purely statistical [3], so that, as far as the calculation of multiplicities is concerned, the set of clusters becomes equivalent, on average, to a large cluster (equivalent global cluster) whose volume is the sum of proper cluster volumes and whose charge is the sum of cluster charges (and thus the conserved charge of the initial colliding system). In such a global averaging process, the equivalent cluster generally turns out to be large enough in mass and volume so that the canonical ensemble becomes a good approximation. In other words, a temperature can be introduced which replaces the $a$ priori more fundamental description in terms of an energy density.

To obtain a simple expression for our further discussion, we neglect for the moment an aspect which is important in any actual analysis. Although in elementary collisions the conservation of the various discrete abelian charges (electric charge, baryon number, strangeness, heavy flavour) has to be taken into account exactly [30], we here consider for the moment a grand-canonical picture. We also assume Boltzmann distributions for all hadrons. The multiplicity of a given scalar hadronic species $j$ then becomes

$$
\left\langle n_{j}\right\rangle^{\text {primary }}=\frac{V T m_{j}^{2}}{2 \pi^{2}} \gamma_{s}^{n_{j}} K_{2}\left(\frac{m_{j}}{T}\right)
$$

with $m_{j}$ denoting its mass and $n_{j}$ the number of strange quarks/antiquarks it contains. Here primary indicates that it gives the number at the hadronisation point, prior to all subsequent resonance decay. The Hankel function $K_{2}(x)$, with $K(x) \sim \exp \{-x\}$ for large $x$, gives the Boltzmann factor, while $V$ denotes the overall equivalent cluster volume. In other words, in an analysis of $4 \pi$ data of elementary collisions, $V$ is the sum of the all cluster volumes at all different rapidities. It thus scales with the overall multiplicity and hence increases with collision energy. A fit of production data based on the statistical hadronisation model thus involves three parameters: the hadronisation temperature $T$, the strangeness suppression factor $\gamma_{s}$, and the equivalent global cluster volume $V$.

As previously discussed, at high energy the temperature turns out to be independent of the initial configuration and this result calls for a universal mechanism underlying the hadronization. In the next paragraph we recall the interpretation of the string breaking as QCD H-U radiation.

2.2. String Breaking and Event Horizon. Let us outline the thermal hadron production process through $\mathrm{H}-\mathrm{U}$ radiation for the specific case of $e^{+} e^{-}$annihilation (see Figure 1). The separating primary $q \bar{q}$ pair excites a further pair $q_{1} \bar{q}_{1}$ from the vacuum, and this pair is in turn pulled apart by the primary constituents. In the process, the $\bar{q}_{1}$ shields the $q$ from its original partner $\bar{q}$, with a new $q \bar{q}_{1}$ string formed. When it is stretched to reach the pair production threshold, a further pair is formed, and so on [31,32]. Such a pair production mechanism is a special case of $\mathrm{H}-\mathrm{U}$ radiation [33-37], emitted 


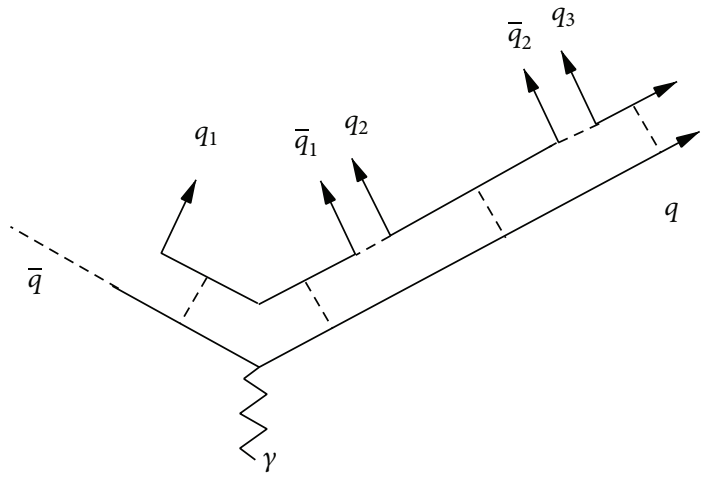

FIGURE 1: String breaking through $q \bar{q}$ pair production.

as hadron $\bar{q}_{1} q_{2}$ when the quark $q_{1}$ tunnels through its event horizon to become $\bar{q}_{2}$. The corresponding hadron radiation temperature is given by the Unruh form $T_{H}=a / 2 \pi$, where $a$ is the acceleration suffered by the quark $\bar{q}_{1}$ due to the force of the string attaching it to the primary quark $Q$. This is equivalent to that suffered by quark $q_{2}$ due to the effective force of the primary antiquark $\bar{Q}$. Hence we have

$$
a_{q}=\frac{\sigma}{w_{q}}=\frac{\sigma}{\sqrt{m_{q}^{2}+k_{q}^{2}}}
$$

where $w_{q}=\sqrt{m_{q}^{2}+k_{q}^{2}}$ is the effective mass of the produced quark, with $m_{q}$ for the bare quark mass and $k_{q}$ the quark momentum inside the hadronic system $q_{1} \bar{q}_{1}$ or $q_{2} \bar{q}_{2}$. Since the string breaks [25] when it reaches a separation distance

$$
x_{q} \simeq \frac{2}{\sigma} \sqrt{m_{q}^{2}+\left(\frac{\pi \sigma}{2}\right)}
$$

the uncertainty relation gives us $k_{q} \simeq 1 / x_{q}$

$$
w_{q}=\sqrt{m_{q}^{2}+\left[\frac{\sigma^{2}}{\left(4 m_{q}^{2}+2 \pi \sigma\right)}\right]}
$$

for the effective mass of the quark. The resulting quark-mass dependent Unruh temperature is thus given by

$$
T(q q) \simeq \frac{\sigma}{2 \pi w_{q}} .
$$

Note that here it is assumed that the quark masses for $q_{1}$ and $q_{2}$ are equal. For $m_{q} \simeq 0$, (5) reduces to

$$
T(00) \simeq \sqrt{\frac{\sigma}{2 \pi}}
$$

as obtained in [25].

If the produced hadron $\bar{q}_{1} q_{2}$ consists of quarks of different masses, the resulting temperature has to be calculated as an average of the different accelerations involved. For one massless quark $\left(m_{q} \simeq 0\right)$ and one of strange quark mass $m_{s}$, the average acceleration becomes

$$
\bar{a}_{0 s}=\frac{w_{0} a_{0}+w_{s} a_{s}}{w_{0}+w_{s}}=\frac{2 \sigma}{w_{0}+w_{s}} .
$$

From this the Unruh temperature of a strange meson is given by

$$
T(0 s) \simeq \frac{\sigma}{\pi\left(w_{0}+w_{s}\right)}
$$

with $w_{0} \simeq \sqrt{1 / 2 \pi \sigma}$ and $w_{s}$ given by (4) with $m_{q}=m_{s}$. Similarly, we obtain

$$
T(s s) \simeq \frac{\sigma}{2 \pi w_{s}}
$$

for the temperature of a meson consisting of a strange quarkantiquark pair $(\phi)$. With $\sigma \simeq 0.2 \mathrm{GeV}^{2},(6)$ gives $T_{0} \simeq$ $0.178 \mathrm{GeV}$. A strange quark mass of $0.1 \mathrm{GeV}$ reduces this to $T(0 s) \simeq 0.167 \mathrm{GeV}$ and $T(s s) \simeq 157 \mathrm{MeV}$, that is, by about $6 \%$ and $12 \%$, respectively.

The scheme is readily generalized to baryons. The production pattern is illustrated in Figure 2 and leads to an average of the accelerations of the quarks involved. We thus have

$$
T(000)=T(0) \simeq \frac{\sigma}{2 \pi w_{0}}
$$

for nucleons,

$$
T(00 s) \simeq \frac{3 \sigma}{2 \pi\left(2 w_{0}+w_{s}\right)}
$$

for $\Lambda$ and $\Sigma$ production,

$$
T(0 s s) \simeq \frac{3 \sigma}{2 \pi\left(w_{0}+2 w_{s}\right)}
$$

for $\Xi$ production, and

$$
T(s s s)=T(s s) \simeq \frac{\sigma}{2 \pi w_{s}}
$$

for that of $\Omega$ 's. We thus obtain a resonance gas picture with five different hadronization temperatures, as specified by the strangeness content of the hadron in question: $T(00)=$ $T(000), T(0 s), T(s s)=T(s s), T(00 s)$ and $T(0 s s)$.

2.3. Strangeness Suppression in Elementary Collisions. In order to evaluate the primary hadron multiplicities, the previous different species-dependent temperatures, fully determined by the string tension $\sigma$ and the strange quark mass $m_{s}$, have been inserted into a complete statistical model calculation [28].

Apart from possible variations of the quantities of $\sigma$ and $m_{s}$, the description is thus parameter-free. As illustration, we show in Table 1 the temperatures obtained for $\sigma=0.2 \mathrm{GeV}^{2}$ and three different strange quark masses. It is seen that in all cases, the temperature for a hadron carrying nonzero 


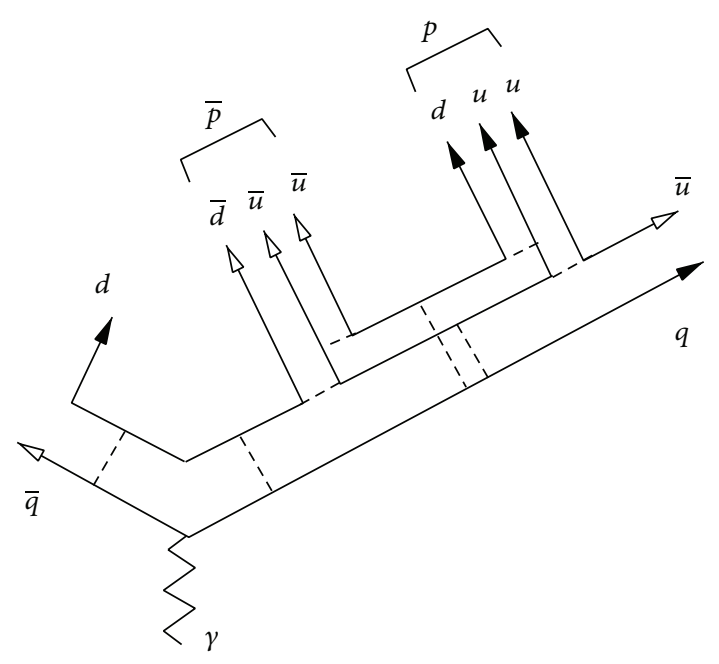

FIGURE 2: Nucleon formation by string breaking through $q \bar{q}$ pair production.

TABLE 1: Hadronization temperatures for hadrons of different strangeness content, for $m_{s}=0.075,0.100,0.125 \mathrm{GeV}$ and $\sigma=$ $0.2 \mathrm{GeV}^{2}$.

\begin{tabular}{lccc}
\hline$T$ & $m_{s}=0.075$ & $m_{s}=0.100$ & $m_{s}=0.125$ \\
\hline$T(00)$ & 0.178 & 0.178 & 0.178 \\
$T(0 s)$ & 0.172 & 0.167 & 0.162 \\
$T(s s)$ & 0.166 & 0.157 & 0.148 \\
$T(000)$ & 0.178 & 0.178 & 0.178 \\
$T(00 s)$ & 0.174 & 0.171 & 0.167 \\
$T(0 s s)$ & 0.170 & 0.164 & 0.157 \\
$T(s s s)$ & 0.166 & 0.157 & 0.148 \\
\hline
\end{tabular}

strangeness is lower than that of nonstrange hadrons and this leads to an overall strangeness suppression in elementary collisions, in good agreement with data [28], without the introduction of the ad-hoc parameter $\gamma_{s}$.

Although a complete statistical hadronization model calculation has been necessary for a detailed comparison with experimental results [28], a rather simple, coarse grain argument can illustrate why a reduction of the $\mathrm{H}-\mathrm{U}$ temperature for strange particle production reproduces the $\gamma_{s}$ effect.

To simplify matters, let us assume that there are only two species: scalar and electrically neutral mesons, "pions" with mass $m_{\pi}$, and "kaons" with mass $m_{k}$ and strangeness $s=1$.

According to the statistical model with the $\gamma_{s}$ suppression factor, the ratio $N_{k} / N_{\pi}$ is obtained by (1) and is given by

$$
\left.\frac{N_{k}}{N_{\pi}}\right|_{\gamma_{s}} ^{\text {stat }}=\frac{m_{k}^{2}}{m_{\pi}^{2}} \gamma_{s} \frac{K_{2}\left(m_{k} / T\right)}{K_{2}\left(m_{\pi} / T\right)}
$$

because there is thermal equilibrium at temperature $T$.

On the other hand, in the H-U based statistical model there is no $\gamma_{s}, T_{k}=T(0 s) \neq T_{\pi}=T(00)=T$ and therefore

$$
\left.\frac{N_{k}}{N_{\pi}}\right|_{\mathrm{H}-\mathrm{U}} ^{\text {stat }}=\frac{m_{k}^{2}}{m_{\pi}^{2}} \frac{T_{k}}{T_{\pi}} \frac{K_{2}\left(m_{k} / T_{k}\right)}{K_{2}\left(m_{\pi} / T_{\pi}\right)} .
$$

Which corresponds to a $\gamma_{s}$ parameter given by

$$
\gamma_{s}=\frac{T_{k}}{T_{\pi}} \frac{K_{2}\left(m_{k} / T_{k}\right)}{K_{2}\left(m_{k} / T_{\pi}\right)} .
$$

For $\sigma=0.2 \mathrm{GeV}^{2}, m_{s}=0.1 \mathrm{GeV}, T_{\pi}=178 \mathrm{Mev}$, and $T_{k}=$ $167 \mathrm{Mev}$ (see Table 1), the crude evaluation by (16) gives $\gamma_{s} \simeq$ 0.73 .

In conclusion, our picture implies that the produced hadrons are emitted slightly "out of equilibrium," in the sense that the emission temperatures are not identical. As long as there is no final state interference between the produced quarks or hadrons, we expect to observe this difference and hence a modification of the production of strange hadrons, in comparison to the corresponding full equilibrium values.

Once such interference becomes likely, due to the increases of parton density, as in high energy heavy ion collisions, equilibrium can be essentially restored producing a small strangeness suppression as we will discuss in the next section.

\section{Hawking-Unruh Strangeness Enhancement in Heavy Ion Collisions}

As previosuly discussed, in elementary collisions the production of hadrons containing $n$ strange quarks or antiquarks is reduced in comparison to nucleus-nucleus scattering and this reduction can be accounted for by the introduction of the phenomenological universal strangeness suppression factor $\gamma_{s}^{n}$.

However, the hadron production in high energy collisions occurs in a number of causally disconnected regions of finite space-time size [38]. As a result, globally conserved quantum numbers (charge, strangeness, and baryon number) must be conserved locally in spatially restricted correlation clusters. This provides a dynamical basis for understanding the suppression of strangeness production in elementary interactions $\left(p p, e^{+} e^{-}\right)$due to a small strangeness correlation volume $[11,30,39,40]$.

The H-U counterpart of this mechanism is that in elementary collisions there is a small number of partons in a causally connected region and the hadron production comes from the sequential breaking of independent $q \bar{q}$ strings with the consequent species-dependent temperatures which reproduce the strangeness suppression.

In contrast, the space-time superposition of many collisions in heavy ion interactions largely removes these causality constraints [38], resulting in an ideal hadronic resonance gas in full equilibrium.

In the H-U approach, the effect of a large number of causally connected quarks and antiquarks can be implemented by defining the average temperature of the system and determining the hadron multiplicities by the statistical model with this "equilibrium" temperature.

Indeed, the average temperature depends on the numbers of light quarks, $N_{l}$, and of strange quarks, $N_{s}$, which, in turn, are counted by the number of strange and nonstrange hadrons in the final state at that temperature. 
A detailed analysis requires again a full calculation in the statistical model that will be done in a forthcoming paper; however the mechanism can be roughly illustrated in the world of "pions" and "kaons" introduced in Section 2.

Let us consider a high density system of quarks and antiquarks in a causally connected region. Generalizing our formulas in Section 2, the average acceleration is given by

$$
\bar{a}=\frac{N_{l} w_{0} a_{0}+N_{s} w_{s} a_{s}}{N_{l} w_{0}+N_{s} w_{s}}
$$

By assuming $N_{l} \gg N_{s}$, by ((6)-(8)), after a simple algebra, the average temperature, $\bar{T}=\bar{a} / 2 \pi$, turns out to be

$$
\bar{T}=T(00)\left[1-\frac{N_{s}}{N_{l}} \frac{w_{0}+w_{s}}{w_{0}}\left(1-\frac{T(0 s)}{T(00)}\right)\right]+O\left[\left(\frac{N_{s}}{N_{l}}\right)^{2}\right]
$$

Now in our world of "pions" and "kaons" one has $N_{l}=2 N_{\pi}+$ $N_{k}$ and $N_{s}=N_{k}$ and therefore

$$
\begin{aligned}
\bar{T}= & T(00)\left[1-\frac{N_{k}}{2 N_{\pi}} \frac{w_{0}+w_{s}}{w_{0}}\left(1-\frac{T(0 s)}{T(00)}\right)\right] \\
& +O\left[\left(\frac{N_{k}}{N_{\pi}}\right)^{2}\right] .
\end{aligned}
$$

On the other hand, in the H-U based statistical calculation the ratio $N_{k} / N_{\pi}$ depends on the equilibrium (average) temperature $\bar{T}$; that is,

$$
\frac{N_{k}}{N_{\pi}}=\frac{m_{k}^{2}}{m_{\pi}^{2}} \frac{K_{2}\left(m_{k} / \bar{T}\right)}{K_{2}\left(m_{\pi} / \bar{T}\right)}
$$

and, therefore, one has to determine the temperature $\bar{T}$ in such a way that (19) and (20) are self-consistent. This condition implies the equation

$$
2 \frac{[1-\bar{T} / T(00)] w_{0}}{[1-T(0 s) / T(00)]\left(w_{s}+w_{0}\right)}=\frac{m_{k}^{2}}{m_{\pi}^{2}} \frac{K_{2}\left(m_{k} / \bar{T}\right)}{K_{2}\left(m_{\pi} / \bar{T}\right)},
$$

that can be solved numerically.

For $\sigma=0.2 \mathrm{GeV}^{2}, m_{s}=0.1$, and the temperatures in Table 1 , the average temperature turns out $\bar{T}=174 \mathrm{Mev}$ and one can evaluate the Wroblewski factor defined by

$$
\lambda=\frac{2 N_{s}}{N_{l}}
$$

where $N_{s}$ is the number of strange and antistrange quarks in the hadrons in the final state and $N_{l}$ is the number of light quarks and antiquarks in the final state minus their number in the initial configuration.

The experimental value of the Wroblewski factor in high energy collisions is rather independent on the energy and is about $\lambda \simeq 0.26$ in elementary collisions and $\lambda \simeq 0.5$ for nucleus-nucleus scattering.
In our simplified world, for $e^{+} e^{-}$annhilation, $\lambda=N_{k} / N_{\pi}$ and is given by (15) with the species-dependent temperatures in Table 1 . One gets $\lambda \simeq 0.26$.

To evaluate the Wroblewski factor in nucleus-nucleus collisions one has to consider the average "equilibrium" temperature $\bar{T}$ and the number of light quarks in the initial configuration. The latter point requires a realistic calculation in the statitical model which includes all resonances and stable particles.

However to show that one is on the right track, let us neglect the problem of the initial configurationa and let us evaluate the effect of substituting in (15) the speciesdependent temperatures with the equilibrium temperature $\bar{T}$. With this simple modification the Wroblewski factor increases, $\lambda=0.33$.

In other terms, the change from a nonequilibrium condition, with species-dependent temperatures, to an equilibrated system with the average temperature $\bar{T}$ is able to reproduce part of the observed growing of the number of strange quarks with respect to elementary interactions.

\section{Conclusions and Comments}

The Hawking-Unruh hadronization mechanism gives a dynamical basis to the universality of the temperature obtained in the statistical model, to the strangeness suppression in elementary collisions and to its enhancement in nucleus-nucleus scatterings.

We are aware that the simplified formulation in Section 3 has to be checked by a full analysis in the hadron resonance gas framework or by montecarlo simulations and that, in addition, the interpretation of production of charmed hadrons in $e^{+} e^{-}$annihilations, which is very successfully described by the hadron resonance gas model with an exact charm conservation, should be addressed through HawkingUnruh radiation mechanism to check if there is a consistent picture.

However the proposed approach is also able to understand the extremely fast equilibration of hadronic matter. Indeed, Hawking-Unruh radiation provides a stochastic rather than kinetic approach to equilibrium, with a randomization essentially due to the quantum physics of the color horizon. The barrier to information transfer due to the event horizon requires that the resulting radiation states excited from the vacuum be distributed according to maximum entropy, with a temperature determined by the strength of the confining field.

The ensemble of all produced hadrons, averaged over all events, then leads to the same equilibrium distribution as obtained in hadronic matter by kinetic equilibration. In the case of a very high energy collision with a high average multiplicity already one event can provide such equilibrium; because of the interruption of information transfer at each of the successive quantum color horizons, there is no phase relation between two successive production steps in a given event. The destruction of memory, which in kinetic equilibration is achieved through sufficiently many successive collisions, is here automatically provided by the tunnelling process. So 
the thermal hadronic final state in high energy collisions is obtained through a stochastic process.

Finally, high energy particle physics, and in particular hadron production, is, in our opinion, the promising sector to find the analogue of the Hawking-Unruh radiation for two main reasons: color confinement and the huge acceleration that cannot be reached in any other dynamical systems.

\section{Conflict of Interests}

The authors declare that there is no conflict of interests regarding the publication of this paper.

\section{References}

[1] F. Becattini, "A thermodynamical approach to hadron production in $e^{+} e^{-}$collisions," Zeitschrift für Physik C, vol. 69, pp. 485492, 1996.

[2] F. Becattini, "Universality of thermal hadron production in $\mathrm{pp}$, $p \bar{p}$ and $e^{+} e^{-}$collisions," in Universality Features in Multihadron Production and the Leading Effect, pp. 74-104, World Scientific, Singapore, 1998, http://arxiv.org/abs/hep-ph/9701275.

[3] F. Becattini and G. Passaleva, "Statistical hadronization model and transverse momentum spectra of hadrons in high energy collisions," European Physical Journal C, vol. 23, no. 3, pp. 551$583,2002$.

[4] F. Becattini and U. Heinz, "Thermal hadron production in pp and $p \bar{p}$ collisions," Zeitschrift für Physik C, vol. 76, no. 268, p. 286, 1997.

[5] J. Cleymans, H. Satz, E. Suhonen, and D. W. Von Oertzen, "Strangeness production in heavy ion collisions at finite baryon number density," Physics Letters B, vol. 242, no. 1, pp. 111-114, 1990.

[6] J. Cleymans and H. Satz, "Thermal hadron production in high energy heavy ion collisions," Zeitschrift für Physik C, vol. 57, pp. 135-147, 1993.

[7] K. Redlich, J. Cleymans, H. Satz, and E. Suhonen, "Hadronisation of quark-gluon plasma," Nuclear Physics A, vol. 566, pp. 391-394, 1994.

[8] P. Braun-Munzinger, J. Stachel, J. P. Wessels, and N. Xu, "Thermal equilibration and expansion in nucleus-nucleus collisions at the AGS," Physics Letters B, vol. 344, no. 1-4, pp. 43-48, 1995.

[9] F. Becattini, M. Gaździcki, and J. Sollfrank, "On chemical equilibrium in nuclear collisions," European Physical Journal C, vol. 5, no. 1, pp. 143-153, 1998.

[10] F. Becattini et al., "Features of particle multiplicities and strangeness production in central heavy ion collisions between $1.7 \mathrm{~A}$ and $158 \mathrm{~A} \mathrm{GeV/c}$, Physical Review C, vol. 64, Article ID 024901, 2001.

[11] P. Braun-Munzinger, K. Redlich, and J. Stachel, "Particle production in heavy ion collisions," in Quark-Gluon Plasma 3, R. C. Hwa and X. N. Wang, Eds., World Scientific, Singapore, 2003.

[12] F. Becattini, "Statistical hadronisation phenomenology," Nuclear Physics A, vol. 702, pp. 336-340, 2001.

[13] J. Letessier, J. Rafelski, and A. Tounsi, "Gluon production, cooling, and entropy in nuclear collisions," Physical Review C, vol. 50, no. 1, pp. 406-409, 1994.

[14] F. Becattini, J. Manninen, and M. Gazdzicki, "Energy and system size dependence of chemical freeze-out in relativistic nuclear collisions," Physical Review C, vol. 73, Article ID 044905, 2006.
[15] P. Braun-Munzinger, D. Magestro, K. Redlich, and J. Stachel, "Hadron production in Au-Au collisions at RHIC", Physics Letters B, vol. 518, no. 1-2, pp. 41-46, 2001.

[16] U. Heinz, "Primordial hadrosynthesis in the little bang," Nuclear Physics A, vol. 661, pp. 140-149, 1999.

[17] R. Stock, "The parton to hadron phase transition observed in $\mathrm{Pb}+\mathrm{Pb}$ collisions at $158 \mathrm{GeV}$ per nucleon," Physics Letters B, vol. 456, no. 2-4, pp. 277-282, 1999.

[18] A. Bialas, "Fluctuations of the string tension and transverse mass distribution," Physics Letters B, vol. 466, no. 2-4, pp. 301304, 1999.

[19] H. Satz, "The search for the QGP: a critical appraisal," Nuclear Physics B-Proceedings Supplements, vol. 94, no. 1-3, pp. 204218, 2001.

[20] J. Hormuzdiar, S. D. H. Hsu, and G. Mahlon, "Particle multiplicities and thermalization in high energy collisions," International Journal of Modern Physics E, vol. 12, no. 5, pp. 649-659, 2003.

[21] V. Koch, "Some remarks on the statistical model of heavy ion collisions," Nuclear Physics A, vol. 715, p. 108, 2003.

[22] L. McLerran, "The quark gluon plasma and the color glass condensate: 4 lectures," http://arxiv.org/abs/hep-ph/0311028.

[23] Y. Dokshitzer, "Historical and futuristic perturbative and nonperturbative aspects of QCD jet physics," Acta Physica Polonica $B$, vol. 36, p. 361, 2005.

[24] F. Becattini, "What is the meaning of the statistical hadronization model?" Journal of Physics: Conference Series, vol. 5, p. 175, 2005.

[25] P. Castorina, D. Kharzeev, and H. Satz, “Thermal hadronization and Hawking-Unruh radiation in QCD," European Physical Journal C, vol. 52, no. 1, pp. 187-201, 2007.

[26] S. W. Hawking, "Particle creation by black holes," Communications in Mathematical Physics, vol. 43, no. 3, pp. 199-220, 1975.

[27] W. G. Unruh, "Notes on black-hole evaporation," Physical Review D, vol. 14, no. 4, pp. 870-892, 1976.

[28] F. Becattini, P. Castorina, J. Manninen, and H. Satz, "The thermal production of strange and non-strange hadrons in $e^{+} e^{-}$ Collisions," European Physical Journal C, vol. 56, no. 4, pp. 493510, 2008.

[29] A. Wroblewski, "On the strange quark suppression factor in high energy collisions," Acta Physica Polonica B, vol. 16, p. 379, 1985.

[30] R. Hagedorn and K. Redlich, "Statistical thermodynamics in relativistic particle and ion physics: canonical or grand canonical?" Zeitschrift für Physik C, vol. 27, pp. 541-551, 1985.

[31] J. D. Bjorken, "Hadron final states in deep inelastic processes," in Current Induced Reactions, vol. 56 of Lecture Notes in Physics, pp. 93-158, Springer, 1976.

[32] A. Casher, H. Neuberger, and S. Nussinov, "Chromoelectricflux-tube model of particle production," Physical Review D, vol. 20, no. 1, pp. 179-188, 1979.

[33] R. Brout, R. Parentani, and P. Spindel, "Thermal properties of pairs produced by an electric field: a tunneling approach," Nuclear Physics B, vol. 353, no. 1, pp. 209-236, 1991.

[34] R. Parentani and S. Massar, "Schwinger mechanism, Unruh effect, and production of accelerated black holes," Physical Review D, vol. 55, no. 6, pp. 3603-3613, 1997.

[35] K. Srinivasan and T. Padmanabhan, "Particle production and complex path analysis," Physical Review D, vol. 60, no. 2, Article ID $024007,1999$. 
[36] D. Kharzeev and K. Tuchin, "From color glass condensate to quark-gluon plasma through the event horizon," Nuclear Physics A, vol. 753, no. 3-4, pp. 316-334, 2005.

[37] S. P. Kim, "Schwinger mechanism and Hawking radiation as quantum tunneling," Journal of the Korean Physical Society, vol. 53, pp. 1095-1099, 2008.

[38] P. Castorina and H. Satz, "Causality constraints on hadron production in high energy collisions," International Journal of Modern Physics E, vol. 23, Article ID 1450019, 2014.

[39] I. Kraus, J. Cleymans, H. Oeschler, K. Redlich, and S. Wheaton, "Chemical equilibrium in collisions of small systems," Physical Review C, vol. 76, Article ID 064903, 2007.

[40] I. Kraus, J. Cleymans, H. Oeschler, and K. Redlich, "Particle production in $p-p$ collisions and predictions for $\sqrt{s}=14 \mathrm{TeV}$ at the CERN Large Hadron Collider (LHC) ", Physical Review C, vol. 79, Article ID 014901, 2009. 

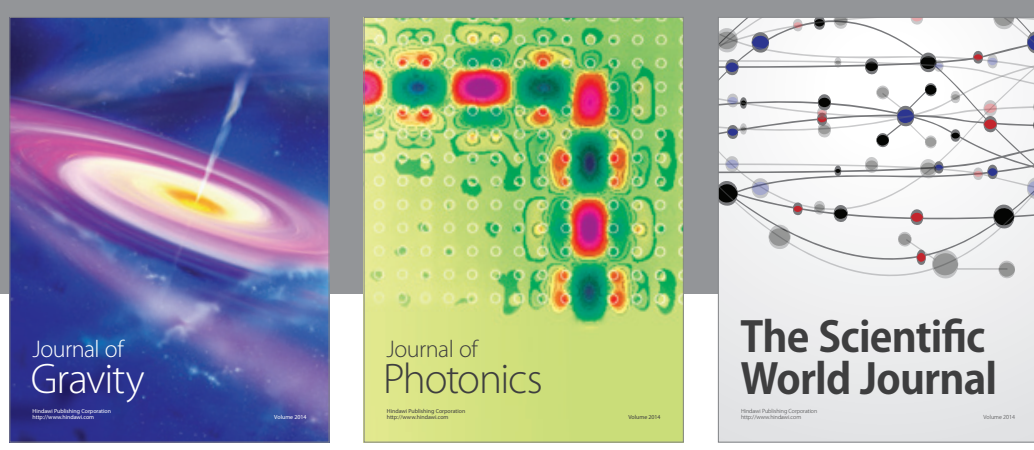

The Scientific World Journal
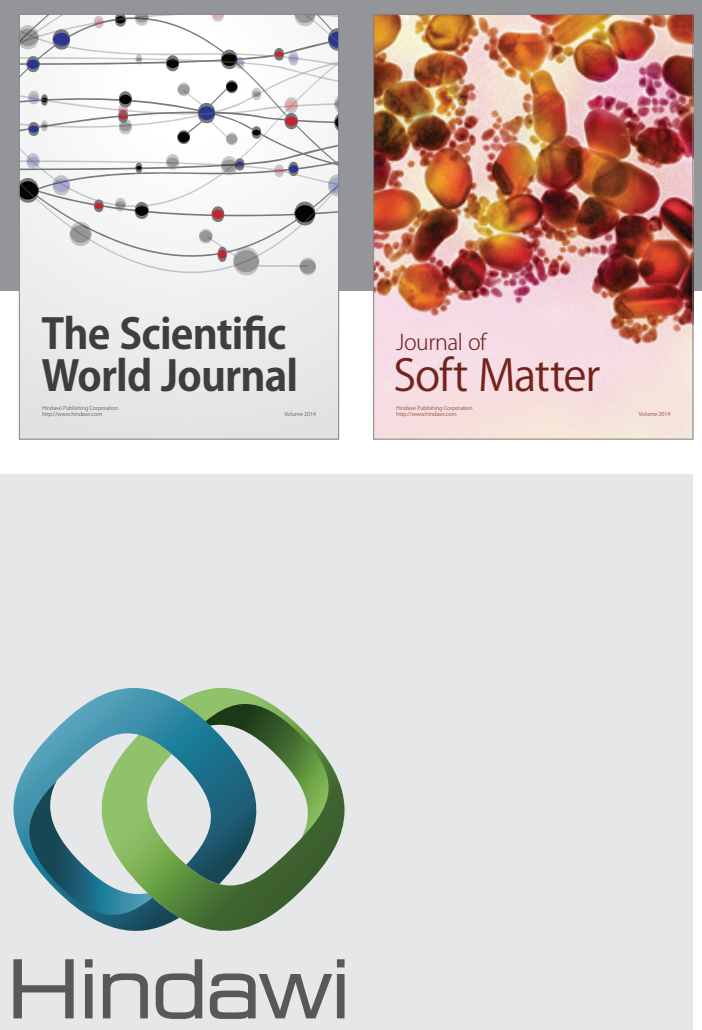

Submit your manuscripts at

http://www.hindawi.com

nternational Journal of

Statistical Mechanics
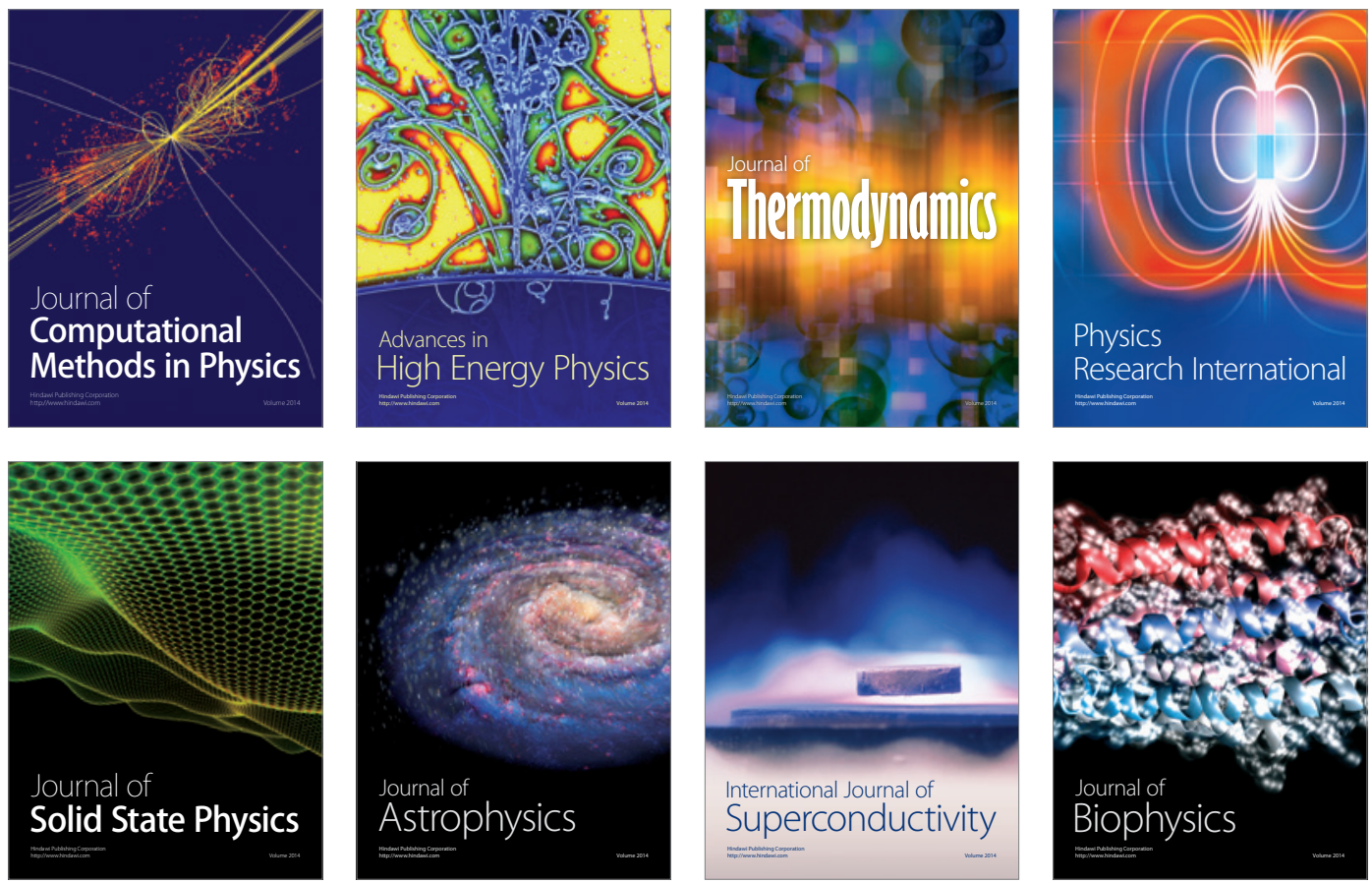
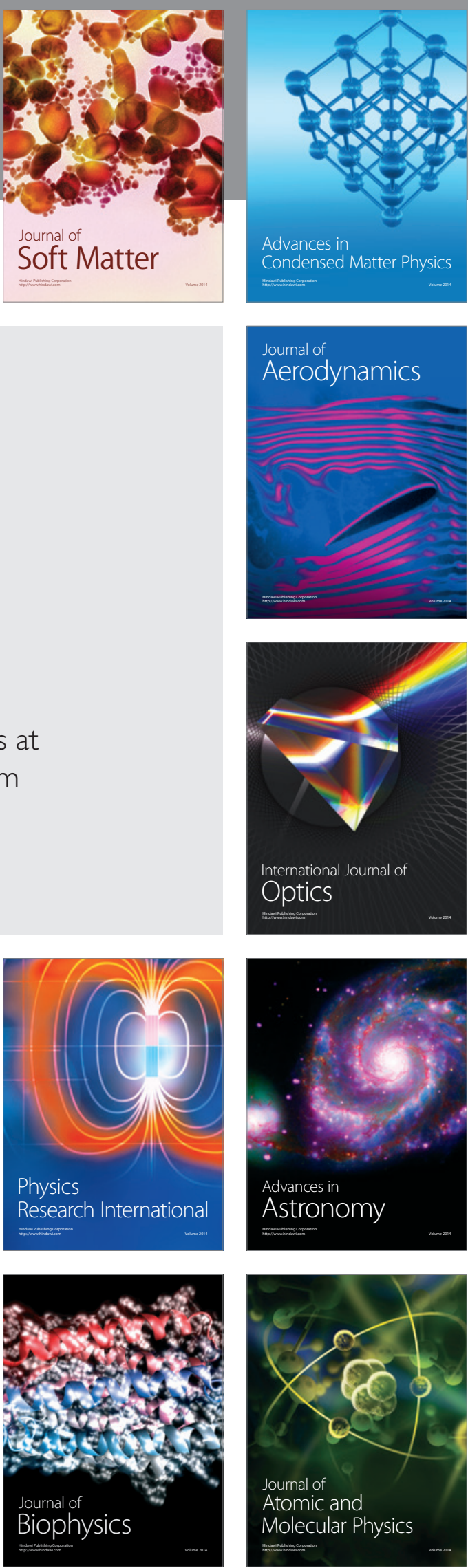